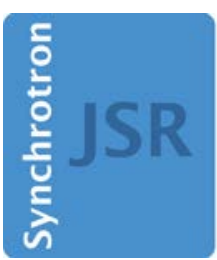

JOURNAL OF

SYNCHROTRON

RADIATION

Volume 26 (2019)

Supporting information for article:

Three-dimensional visualization of fibrous tissues in cirrhotic rats via X-ray phase-contrast computed tomography with iodine staining

Jianbo Jian, Xinyan Zhao, Lili Qin, Yuqing Zhao, Mengyu Sun, Wenjuan Lv and Chunhong $\mathrm{Hu}$ 


\section{S1. Optimization of the staining time}

For stain development and optimization, the staining time from 1 to 7 days was tested in this study. The criteria for a complete iodine penetration were: (i) a homogeneous contrast within the structural regions of the soft tissue without the occurrence of unstained areas, and (ii) a negative contrast only for the fibrous tissues and blood vessels. Figure S1 presented part results of the test. The results showed that (i) vessels could be clearly seen, but the fibrous tissues lacked sufficient contrast at 1 days (Fig. S1A); (ii) the fibrous tissues could be seen but lacked obvious margins at 3 days (Fig. S1B); (iii) a homogeneous contrast within the structural regions of the soft tissue could be seen and obvious margins of the fibrous tissues could be clearly distinguished at 5 days (Fig. S1C), confirmed by the histological section (Fig. S1D); (iv) the fibrous tissues were over diffused, and parts of the structure could not be clearly seen at 7 days (Fig. S1E). The results showed that 5 days met the criteria and was the optimal time at which the liver parenchyma was fully stained and iodine does not diffuse into the fibrous tissues.
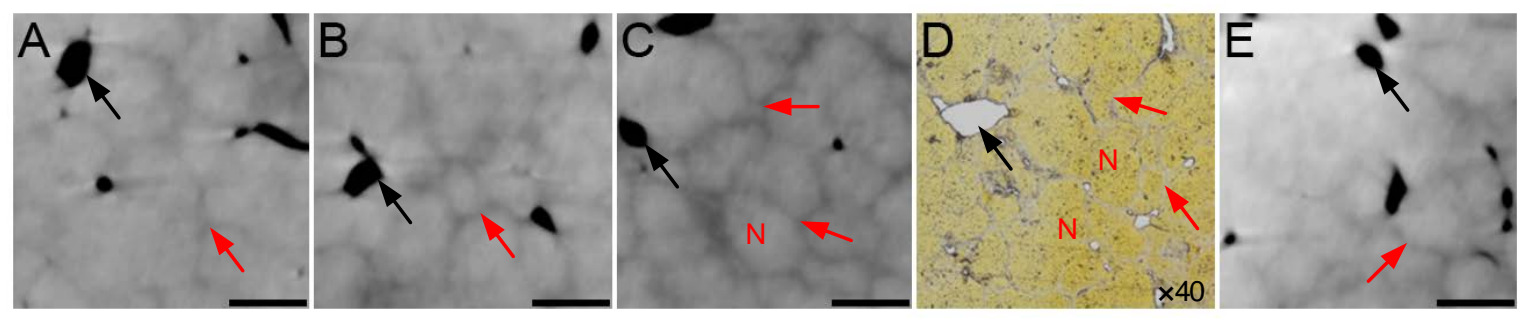

Figure S1 Optimization of the staining time. A, B, C and E, PCCT slices of samples with staining time at 1, 3, 5 and 7 days, respectively. D, Histological section (without any histological staining) of the sample with staining time at 5 days. As the histological section shown, except fibrosis tissues (red arrows) and vessels (black arrows), the liver parenchyma (yellow color) was fully stained at the staining time. The high atomic number $(Z=53)$ component generates a particularly high-contrast enhancement for X-rays. A higher accumulation of iodine within the liver parenchyma generates a strongly negative contrast for the fibrosis tissues, allowing for the visualization of fibrosis tissues, small nodules $(\mathrm{N})$ and vessels in the X-rays images at the same time. Scalebar $=500 \mu \mathrm{m}$. 


\section{S2. Process of CT Reconstruction and 3D Visualization}

The complete process of the image reconstruction is provided in Fig. S2 as follows.

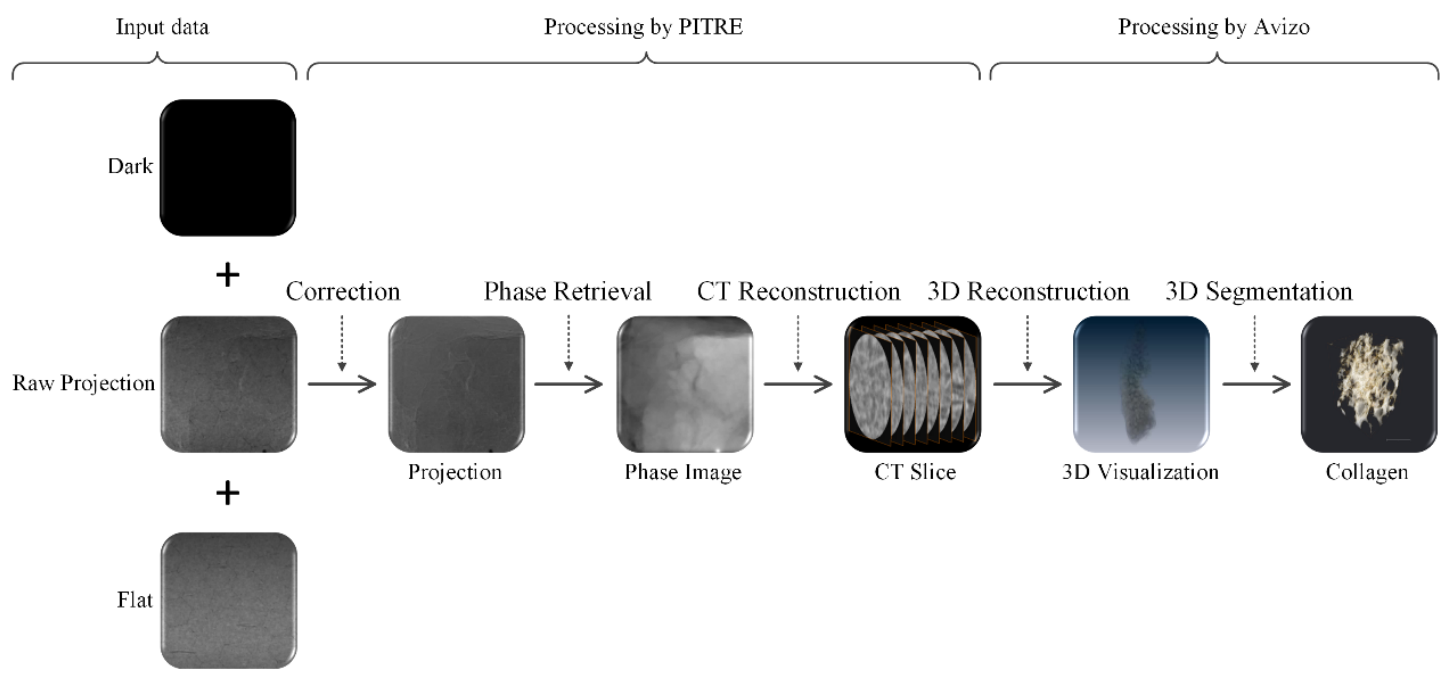

Figure S2 Schematic sketch of the image reconstruction and 3D visualization. The obtained raw projections were first subjected to flat-field and dark-field corrections, and then a phase retrieval method (PAD-BA) was employed to extract phase information from the projections after the corrections. Subsequently, the phase images were reconstructed using the filtered back-projection (FBP) algorithm to build a stack of CT slices. The 3D reconstruction of the HCC tissues was performed with the imageprocessing software Avizo Fire 8.0. Threshold segmentation and surface reconstruction were used in the reconstruction of the 3D model. Finally, the 3D visualization of the microstructures in cirrhotic tissues was achieved after the 3D segmentation. 


\section{S3. Image Analysis}

\section{S3.1. Fibrosis Area}

Fibrosis area has been widely used in staging cirrhosis. In this study, areas of fibrosis in the histopathologic sections were stained by Sirius Red and assessed in five regions of interest (ROIs) for each sample (30 ROIs in total) at 100× magnification. To ensure equal ROI size and location on the PCCT image, the ROI from the histological image was copied and subsequently pasted on the corresponding PCCT image by keeping the exact same position. The fibrosis area was expressed as a relative percentage between the area of fibrosis and the total area of the ROI, and analyzed using ImagePro Plus 6.0 software (Media Cybernetics, Bethesda, Maryland, USA).

\section{S3.2. Septal Width}

Five histopathologic sections (original magnification, $\times 100$; Sirius Red staining) and the corresponding PCCT images from each sample were selected as ROIs to assess the width of fibrous septa. A position of the fibrous septa was measured on each ROI, and 30 measurements in total would be acquired using Image-Pro Plus 6.0 software. To ensure equal location on the PCCT image, the position of the septal width from the pathological image was marked and subsequently copied to the corresponding PCCT image by keeping the exact same position (Fig. S3).

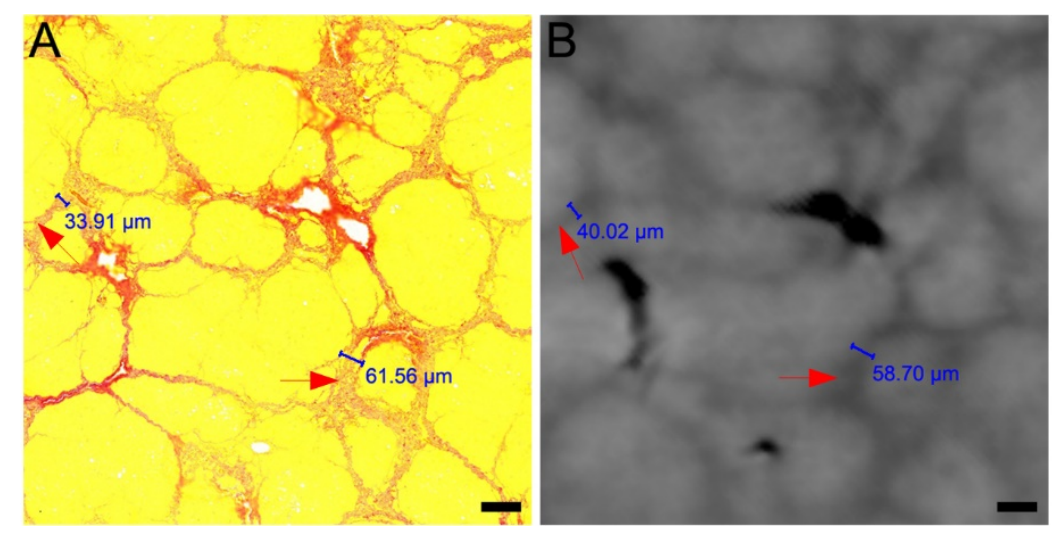

Figure S3 Measurement of the septal width. A, Histopathologic section (original magnification, $\times 100$ ) stained with Sirius Red. $B$, The corresponding PCCT image. Fibrous septa (red arrows) are clearly visualized in the PCCT image $(B)$ which has a close resemblance to the histological section $(A)$. The widths of the fibrous septa (blue lines) at the same position in $(A)$ and $(B)$ are measured via ImagePro Plus 6.0 software, and the results are shown in the figures. Scale bar $=100 \mu \mathrm{m}$. 


\section{S3.3. Nodular Size}

The small nodules were verified by the histopathologic sections stained by Sirius Red at 100x magnification. Nodular sizes to be measured in the histopathologic and corresponding PCCT images were marked with same symbol $(\mathrm{N})$ in Fig. S4. Five nodular sizes were acquired from each sample and 30 measurements were finally obtained via Image-Pro Plus 6.0 software.

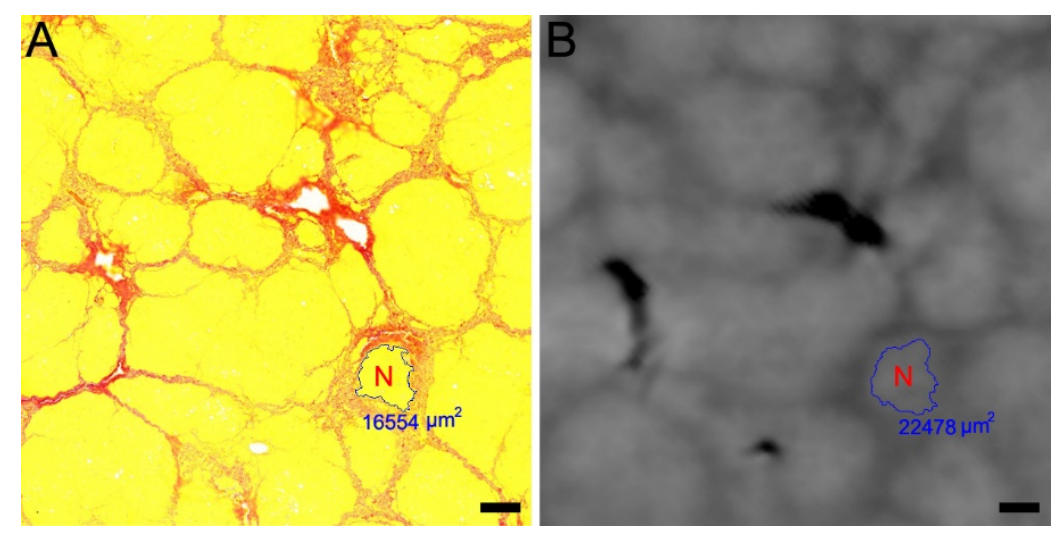

Figure S4 Measurement of the nodular size. A, Histopathologic section (original magnification, $\times 100$; Sirius Red staining). $B$, The corresponding PCCT image. PCCT image $(B)$ clearly presents the small nodules $(\mathrm{N})$ surrounded by fibrous septa, confirmed by the corresponding histopathologic section $(A)$. The area of the nodules (blue curves) at the same position in $(A)$ and $(B)$ are measured via ImagePro Plus 6.0 software, and the nodular size is approximately $1.66 \times 10^{4} \mu \mathrm{m}^{2}$ in $(A)$ and $2.25 \times 10^{4} \mu \mathrm{m}^{2}$ in (B). Scale bar $=100 \mu \mathrm{m}$.

\section{S4. 3D Quantitation of the Fibrous Tissues}

In 2D images, the quantity of fibrosis (fibrosis area) was expressed as a relative percentage between the area of fibrosis and the total area of the ROI Therefor, the fibrosis volume defined as a relative percentage between the volume of fibrosis and the total volume of the region of interest (VOI) was used to assess the fibrous tissues in 3D images. In this study, five non-overlapping VOIs of $200 \times 200 \times 200$ pixels for each sample (30 VOIs in total) selected to assess the fibrosis volume. The 3D quantitation was performed via the Avizo Fire 8.0 software. The results showed the fibrosis volume of the cirrhotic livers was $10.6 \pm 0.9 \%$. which was a little different from the findings of fibrosis area in the 2D images. Compared with the fibrosis area, fibrosis volume which was measured based on the 3D data may be more accurate and comprehensive. The fibrosis volume is a primary trial to explore the potential of this method in 3D quantitative evaluation of the fibrous tissues in cirrhosis, which needs further studies to confirm the results. With further development of 3D imaging techniques, 3D quantitative evaluation of the fibrous tissues will be more widely used in the future. 